\title{
Ureteric Bud-derivatives in Wilms Tumor and Nephrogenic Rest
}

\author{
BEATRIX SARKANY ${ }^{1}$, GYULA KOVACS ${ }^{1,2}$ and DANIEL BANYAI ${ }^{1}$ \\ ${ }^{1}$ Department of Urology, Medical School, University of Pecs, Pecs, Hungary; \\ ${ }^{2}$ Medical Faculty, Ruprecht-Karls-University, Heidelberg, Germany
}

\begin{abstract}
Background/Aim: Recent studies suggest that not only the nephrogenic blastema but also the ureteric bud is involved in oncogenesis of Wilms' tumor (WT). However, the occurrence of ureteric bud $(U B)$ derivatives in nephrogenic rest is not yet known. The aim of our study was to find $U B$ derivatives in WT. Materials and Methods: Keratin 17 (KRT17) is expressed exclusively in UB in foetal kidneys. In this study KRT17 immunohistochemistry was used to detect UB-derivatives in 21 triphasic, 2 stromal and 3 epithelial predominant WTs and 9 nephrogenic rests. Results: We have detected KRT17 positive tubular structures resembling UB in 3 of 9 nephrogenic rests and 15 of 26 WTs. Conclusion: Not only the metanephric blastema but also the UB is involved in the histogenesis of nephrogenic rest and WT.
\end{abstract}

Wilms' tumor (WT) or nephroblastoma is suggested to develop from maturation-arrested nephrogenic blastemal cells $(1,2)$. However, in triphasic WTs the presence of ureteric bud (UB)-like tubular structures surrounded by blastemal cells appear to indicate the involvement of UB or UB-derivatives in the development of WTs (3). A recent global gene expression analysis uncovered genes expressed in distinct compartments of the developing kidney as well as in WT (4). The presence of UB-like epithelial structures in WT has been confirmed by immunohistochemistry $(3,4)$. Recently, we showed that KRT17 expression is restricted to cells of the UB in foetal kidneys and, therefore, it can be used as biomarker to detect UB derivatives (5). To obtain more information on the involvement of $\mathrm{UB}$ in the

This article is freely accessible online.

Correspondence to: Danial Banyai, Department of Urology, Medical School, University of Pecs, Munkacsy M. u. 2, H-7621 Pecs, Hungary. Tel: +36 72507334, Fax: +36 72242374, e-mail: banyai.daniel@pte.hu

Key Words: Ureteric bud, Wilms'tumor, nephrogenic rest, keratin 17 , immunohistochemistry. histogenesis of WT and its precursor lesions, we analysed the expression of KRT17 in WTs and accompanying nephrogenic rests by immunohistochemistry.

\section{Materials and Methods}

Tissue samples. Paraffin embedded material of foetal kidneys, WTs and nephrogenic rests were obtained from the Institute of Pathology, Medical School, University of Pecs in Hungary. We included foetal kidneys of $10,12,15,17$ and 21 weeks of gestation, 21 tri- or biphasic WT, 3 cases with blastemal, another 3 cases with epithelial and two with stromal predominant histological pattern. We have analysed 9 perilobar nephrogenic rests (PLNR) of 2 to $5 \mathrm{~mm}$ in diameter, including two hyperplastic PLNR for the expression of the KRT17 protein. The use of tissue samples for this study was approved by the Ethics Committee of the University Pecs, Hungary (No. 5343/2014).

Immunohistochemistry. Following devaxing and rehydration, the $4 \mu \mathrm{m}$-thick sections were subjected to heat-induced epitope retrieval in citrate buffer, pH 6.0 in 2100-Retriever (Pick-Cell Laboratories, Amsterdam, The Netherlands). Endogenous peroxidase activity was blocked using the Envision FLEX Peroxydase Blocking Reagent (DAKO, Glostrup, Denmark) for $10 \mathrm{~min}$ at room temperature. The tissue sections were incubated in a moist chamber for $1 \mathrm{~h}$ at room temperature with a rabbit polyclonal anti-KRT17 antibody (HPA 000452, lot Nr. A08686, Sigma Aldrich, Budapest, Hungary) in a 1:500 dilution. EnVision FLEX horse-radish-peroxydase-conjugated secondary antibody (DAKO) was applied for 30 minutes at room temperature. The signal was visualized using Amino-ethyl-carbazol (AEC) (DAKO). Tissue sections were counterstained with Mayer's haematoxylin (Lillie's modification, DAKO) and after $10 \mathrm{~s}$ bluing in ammonium-hydroxide solution, mounted in Glycergel (DAKO). As a negative control the primary antibody was omitted. Photographs were taken using a Leitz DMRBE microscope, equipped with HC PLAN APO 20x0.70 objective, and a ProgRes C14 camera (Leitz, Wetzlar, Germany).

\section{Results}

Expression of KRT17 in foetal kidney. We detected the expression of KRT17 in ureteric bud and emerging collecting duct in foetal kidneys at 10 weeks of gestation (Figure 1A and B). Each cell of the ureteric bud and medullary 
collecting duct displayed KRT17 staining. No expression was seen in metanephric mesenchyma-derived structures, such as condensed blastemal cells, renal vesicles or the Sshaped body.

Expression of KRT17 in PLNR and in WT. Three of the 9 PLNR displayed KRT17-positive tall columnar tubules embedded in KRT17-negative nephrogenic rests (Figure 1C and D). KRT17 positive tubular structures were detected in 13 out of the 21 triphasic WTs. UB-derived tubules lined with tall columnar epithelial cells displaying strong KRT17 staining were embedded in undifferenctiated blastema (Figure 1E). Some of the dilated tubules with cuboidal epithelial cells were also positive for the KRT17 antibody (Figure 1F). We also found UB-derived tubules lined with KRT17 positive high columnar cells in stromal predominant WT (Figures 1G). In some areas of predominantly stromal WT, a group of UB-derivative tubules mixed with renal vesicle-like KRT17 negative tubules were seen (Figure H). In epithelial predominant WT all epithelial tubular structures were consequently negative for KRT17 immunohistochemistry (Figure 1I).

\section{Discussion}

Kidney develops from cells of the metanephric mesenchyma as a result of inductive interaction between the metanephric blastema and UB (6). The former differentiates into epithelial structures of nephron from the proximal towards the distal convoluted tubules and forms the stromal components of the kidney. The UB gives rise to the connecting tubules and the collecting ducts of the kidney. WT has been shown to develop due to arrested differentiation of the metanephric blastema, resulting in tumorigenic growth of blastemal, epithelial and stromal cells. The three elements can be seen in WT with classical triphasic histology, even though epithelial, blastemal or stromal predominant variants may also occur $(2,7)$.

The exclusive role of metanephric blastema in the development of WT is challenged by Stupar et al., showing UB-derivative tubular structures in triphasic WTs (3). A recent comparative gene expression study selected genes expressed preferentially in the developing kidney and in WT and confirmed the occurrence of UB-like epithelial structures in triphasic WT using immunohistochemistry (4). Based on these two observations it appears that both the metanephric blastema and the UB are involved in the oncogenesis of WT.

Wilms tumor is suggested to develop from nephrogenic rest, which frequently accompanies clinically manifested WT $(1,7)$. Despite this, the possible involvement of the ureteric bud in the histogenesis of nephrogenic rests is not yet known. We showed in this study that KRT17 positive tubules resembling UB-derivative cells can already be seen in WT precursors, in nephrogenic rests.
KRT17, a 46-47 kDa type I keratin supports the integrity and mechanical stability of epithelial cells, regulates protein synthesis via intracellular signaling pathways and protects tubular cells from apoptosis (8-11). The role of KRT17 in growth and regular branching of the ureteric bud in foetal kidney is unknown. In this study we used KRT17 as marker to recognize UB-derived epithelial structures in nephrogenic rests and WTs.

In summary, we showed that KRT17, which is known to be expressed in multilayered epithelium, is expressed in single layered epithelium of UB in foetal kidney. We also documented that UB-derivatives occur not only in WT but also in nephrogenic rests. We suggest that the causality of WT should include in addition to the maturation-arrested nephrogenic blastemal cells a possible role for the ureteric bud cells. Further studies will help delineate this process.

\section{Conflicts of Interest}

The Authors have no conflicts of interest to declare.

\section{Authors' Contributions}

BS and DB designed the research study, BS performed the immunohistochemistry, BS and GK analyzed the data, BS and DB wrote the manuscript and GK reviewed the manuscript. All Authors read and approved the final version of the manuscript.

\section{Acknowledgements}

This work was supported by a Grant of the Medical Faculty, University of Pecs, Hungary to Daniel Banyai (PTE-AOK-KA-2018/16).

Figure 1. Expression of the KRT17 protein in foetal kidneys, nephrogenic rests and Wilms' tumors. The background was stained with Mayer's haematoxylin. A) KRT17 (brown staining) expressed exclusively in utereric bud $(U B)$. No positive immunreaction was seen in surrounding blastemal cells and S-shaped body (SSB). B) Strong KRT17 immunreaction in each cell of the medullary collecting duct (arrows). C) Tubular cells of a perilobar nephrogenic rests are negative for KRT17, but some UB-derived tubules display KRT17 positivity (arrows). Differentiated cortical collecting ducts also show a week KRT17 taining (short arrows). D) A hyperplastic perilobular nephrogenic rest negative for KRT17 staining but larger tubules with columnar epithels show positive KRT17 staining (arrows). E) A single UB-derivative tubulus lined by tall columnar cells display strong KRT17 staining (star). It is surrounded with tumorous blastemal cells resembling induction/aggregation phase around the UB in embryonal kidneys. F) Dilated UB-derived tubulus (star) surrounded by blastemal cells. G) Two UB-like tubules lined with KRT17 positive tall columnar cells (star) embedded in fibroblastic stroma. H) A group of epithelial tubules, two of them with KRT17 positive cells surrounded with stromal differentiated blastemal cells. I) Smaller and larger tubules, some resembling renal vesicles in epithelial predominant Wilms' tumor. Lack of KRT17 staining indicates their blastemal origin. A-I the same magnification, Scale bar: $35 \mu \mathrm{m}$. 

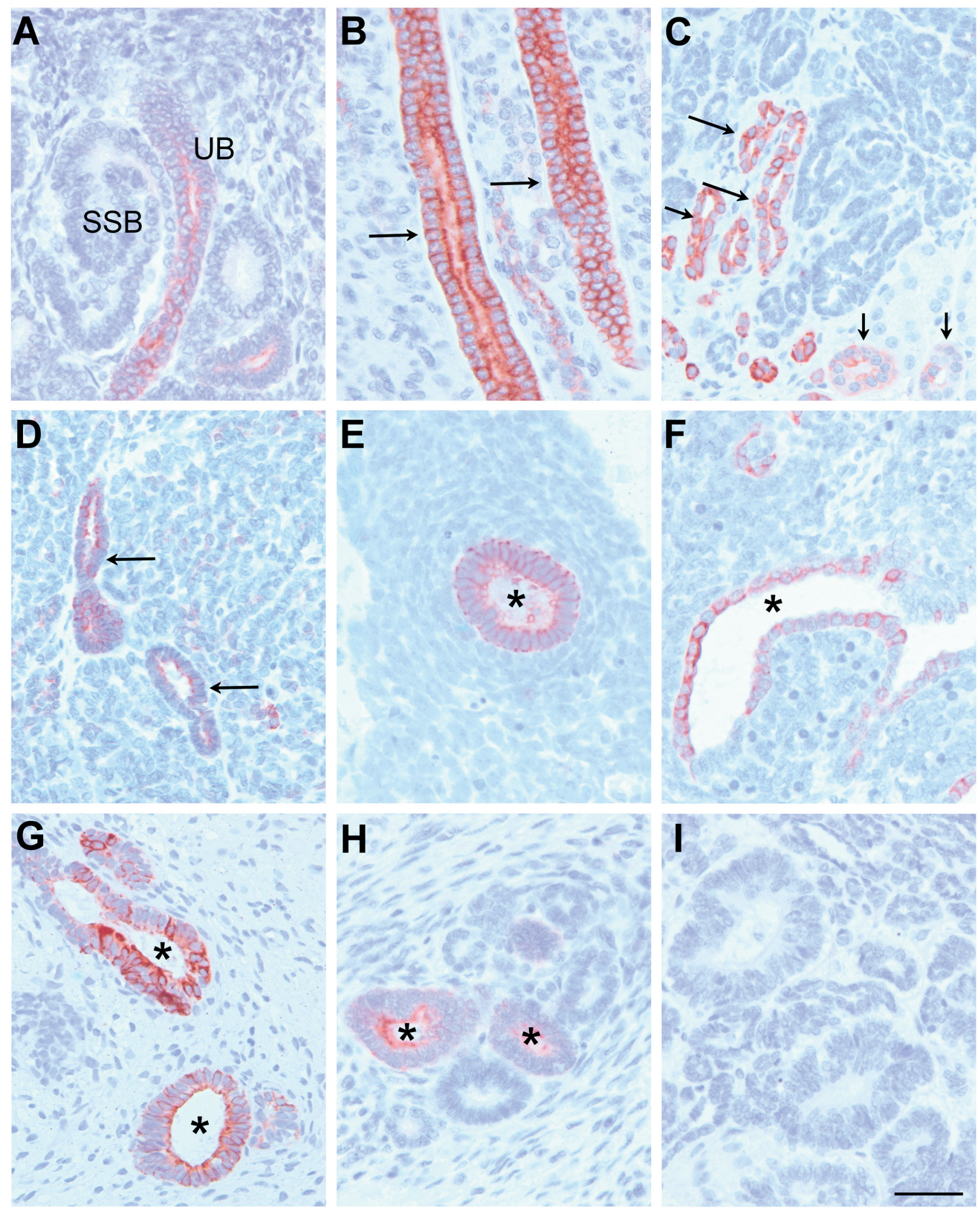


\section{References}

1 Beckwith JB, Kiviat NB and Bonadio JF: Nephrogenic rests, nephroblastomatosis, and the pathogenesis of Wilms' tumor. Pediatr Pathol 10(1-2): 1-36, 1990. PMID: 2156243. DOI: $10.3109 / 15513819009067094$

2 Moch H, Cubilla AL, Humphrey PA, Reuter VE and Ulbright TM: The 2016 WHO classification of tumours of the urinary system and male genital organs-part A: renal, penile, and testicular tumours. Eur Urol 70(1): 93-105, 2016. PMID: 26935559. DOI: $10.1016 /$ j.eururo.2016.02.029

3 Stupar Z, Chi S, Veszpremi B, Koesters R, Stallmach T, Geng JG and Kovacs G: Wilms' tumour may also develop from impaired differentiation of the ureteric bud. Histopathology 51(2): 265-268, 2007. PMID: 17650220. DOI: 10.1111/j.13652559.2007.02741.x

4 Fukuzawa R, Anaka MR, Morison IM and Reeve AE: The developmental programme for genesis of the entire kidney is recapitulated in Wilms tumour. PLoS One 12(10): e0186333, 2017. PMID: 29040332. DOI: 10.1371/journal.pone.0186333

5 Sarlos DP, Yusenko MV, Peterfi L, Szanto A and Kovacs G: Dual role of KRT17: development of papillary renal cell tumor and progression of conventional renal cell carcinoma. J Cancer 10(21): 5124-5129, 2019. PMID: 31602265. DOI: 10.7150/jca.32579

6 Little MH: Improving our resolution of kidney morphogenesis across time and space. Curr Opin Genet Dev 32: 135-143, 2015. PMID: 25819979. DOI: 10.1016/j.gde.2015.03.001
7 Beckwith JB: Nephrogenic rests and the pathogenesis of Wilms tumor: developmental and clinical considerations. Am J Med Genet 79(4): 268-273, 1998. PMID: 9781906. DOI: 10.1002/(sici)10968628(19981002)79:4<268::aid-ajmg7>3.0.co;2-i

8 Moll R, Divo M and Langbein L: The human keratins: biology and pathology. Histochem Cell Biol 129(6): 705-733, 2008. PMID: 18461349. DOI: 10.1007/s00418-008-0435-6

9 Tong X and Coulombe PA: Keratin 17 modulates hair follicle cycling in a TNFalpha-dependent fashion. Genes Dev 20(10): 1353-1364, 2006. PMID: 16702408. DOI: 10.1101/gad.1387406

10 Kim S, Wong P and Coulombe PA: A keratin cytoskeletal protein regulates protein synthesis and epithelial cell growth. Nature 441(7091): 362-365, 2006. PMID: 16710422. DOI: 10.1038/nature 04659

11 Yang L, Zhang S and Wang G: Keratin 17 in disease pathogenesis: from cancer to dermatoses. J Pathol 247(2): 158165, 2019. PMID: 30306595. DOI: 10.1002/path.5178

Received January 25, 2021 Revised April 8, 2021 Accepted April 15, 2021 